\title{
ACTAS DEL CURSO "CLAVES PARA AFRONTAR RIESGOS LABORALES EMERGENTES: EL FUTURO DE LA PREVENCIÓN"
}

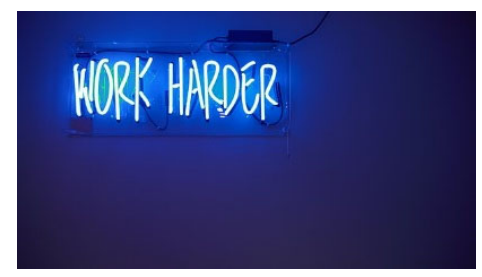

SUSANA RODRÍGUEZ ESCANCIANO

Catedrática de Derecho del Trabajo y de la Seguridad Social

HENAR ÁLVAREZ CUESTA

Profesor Titular de Derecho del Trabajo y de la Seguridad Social

Universidad de León

Durante los días 23, 24, 25 y 26 de septiembre de 2019 de 16:00 a 20:00 horas, en las Facultades de Derecho y Ciencias del Trabajo de la Universidad de León tuvo lugar el Curso titulado "Claves para afrontar riesgos laborales emergentes: el futuro de la prevención", dirigido por las Profesoras Susana Rodríguez Escanciano y Henar Álvarez Cuesta y subvencionado por la Junta de Castilla y León al amparo de la Orden $\mathrm{EMP} / 278 / 2018$, de 8 de marzo.

En el inicio del curso intervino en primer lugar el Prof. Juan Carlos Álvarez Cortés, TU Derecho del Trabajo y de la Seguridad Social de la Universidad de Málaga, tratando el tema de los "Riesgos vinculados a la digitalización de las empresas"; a continuación el Prof. David Montoya Medina, TU Derecho del Trabajo y de la Seguridad Social de la Universidad de Alicante, habló sobre "Riesgos vinculados a la automatización de los procesos e incorporación de Inteligencia Artificial". Los "Riesgos vinculados a la biotecnología" fueron analizados por el Prof. Federico de Montalvo Jääskeläinen, TU de Derecho Constitucional de la Universidad Pontificia de Comillas, presidente del Comité de Bioética de España y miembro del Comité Internacional de Bioética de la Unesco (International Bioethics Committee of UNESCO, IBC). Para finalizar, el Catedrático de Derecho del Trabajo y de la Seguridad 
Social de la Universidad de León, Juan José Fernández Domínguez, abordó los "Riesgos psicosociales vinculados a la sociedad tecnológica".

En la segunda sesión, el Prof. Javier Fernández-Costales Muñiz, TU de Derecho del Trabajo y de la Seguridad Social de la Universidad de León examinó los "Riesgos vinculados a las fuentes de energía renovables y reciclaje"; y las Directoras del curso disertaron sobre "Riesgos vinculados a las nuevas formas de trabajo agropecuario" (Henar Álvarez Cuesta) y "Riesgos vinculados a la construcción ecológica" (Susana Rodríguez Escanciano). Por último, los "Riesgos vinculados a las nuevas formas de movilidad sostenible" fueron abordados por el Prof. Rodrigo Tascón López, TU de Derecho del Trabajo y de la Seguridad Social de la Universidad de León.

En la tercera, se analizó el impacto de los riesgos emergentes en los colectivos más vulnerables. Así, el Prof. Roberto Fernández Fernández, TU de Derecho del Trabajo y de la Seguridad Social de la Universidad de León habló sobre "La formación inicial en materia preventiva para trabajadores jóvenes y para los inmigrantes con escasa experiencia laboral"; la Profa María de los Reyes Martínez Barroso, Catedrática de Derecho del Trabajo y de la Seguridad Social de la Universidad de León analizó "La necesidad de recualificación en materia preventiva de los trabajadores maduros"; la Profa. Beatriz Agra Viforcos, TU de Derecho del Trabajo y de la Seguridad Social de la Universidad de León abordó el estudio de "Las mujeres ante la brecha digital: especial atención a los riesgos en materia preventiva"; y el Prof. José Gustavo Quirós Hidalgo, TU de Derecho del Trabajo y de la Seguridad Social de la Universidad de León finalizó tratando "La potencialidad de los cambios tecnológicos para la incorporación segura al mercado de trabajo de las personas con discapacidad".

En la última jornada se realizaron dos Mesas redondas, la primera de ellas sobre "Soluciones para un adecuado diseño de la prevención de riesgos en el talento sin fronteras y las nuevas vías de emprendimiento" en la que intervinieron Natalia Ordóñez Pascua, Profa. Ayudante Doctor de Derecho del Trabajo y de la Seguridad Social de la Universidad de León analizando la "Prevención de riesgos y talento sin fronteras"; e Prof. F. Xabiere Gómez García, Contratado Predoctoral en formación de Derecho del Trabajo y de la Seguridad Social de la Universidad de León sobre "La prevención de riesgos en trabajadores desplazados"; y la Profa. Cristina González Vidales, Contratada Predoctoral en formación de Derecho del Trabajo y de la Seguridad Social de la Universidad de León sobre "Situación de la Prevención Laboral en el Emprendimiento Digital".

En la segunda mesa se abordó por parte de los agentes sociales "El futuro de la prevención de riesgos laborales en Castilla y León desde la perspectiva de los interlocutores sociales: una apuesta por el diálogo social". En ella intervinieron Dña Sheila Mateo Canelo, Representante de CC OO; Dña. Azucena Pérez Álvarez, Representante de UGT; D. José María Barrios Manso, Representante CSIF y Dña. Marta Uriarte Laine, Representante de FELE. En dicha mesa se puso de relieve las diferentes actuaciones que en el marco del diálogo social y en la materia preventiva 
específica estaban realizando los agentes sociales y la Administración Pública autonómica, y se subrayó la importancia que la prevención de los riesgos ha de tener a la hora de llevar a cabo procesos de digitalización y automatización.

El objetivo fundamental de dicho curso consistió en la difusión y transmisión a los asistentes de un conocimiento completo y detallado de los desafíos que plantea la prevención de riesgos laborales en un sistema productivo cada vez más digitalizado y automatizado y el impacto que suponen los distintos cambios operados tanto en ciertos sectores de capital importancia en la Comunidad Autónoma (construcción, agricultura, ganadería, producción energética o reciclaje), como en determinados colectivos, como trabajadores maduros, jóvenes, mujeres, o personas con discapacidad.

El interés de cada ponencia y la actualidad de los temas tratados quedan reflejados no solo en las cifras de asistencia y en el debate producido durante su realización, sino también en los resúmenes de cada intervención ofrecidos a continuación (disponibles también en el Repositorio de acceso abierto de la Universidad de León).

RIESGOS VINCULADOS A LA DIGITALIZACIÓN DE LAS EMPRESAS

\section{Dr. Juan Carlos Álvarez Cortés}

Universidad de Málaga

La aparición de nuevas tecnologías, como el internet de las cosas (IdC - IoT), la Inteligencia Artificial, los macrodatos, la computación en la nube, la robótica colaborativa, la Realidad Aumentada, la fabricación aditiva y las plataformas de internet, tiene y va a tener un profundo impacto en el mundo laboral.

Aunque la aplicación de las tecnologías habilitadas por las tecnologías de la información y comunicación (TIC) se encuentra actualmente en distintos grados de difusión, según sectores de actividad, lo cierto es que en todos ellos se están integrando de forma acelerada ya que permiten una mayor productividad, ahorrando costes tras el período de amortización de la inversión tecnológica y aumentando las posibilidades de obtener beneficios.

Es más que posible que a lo largo de los próximos años próximos puedan producirse cambios significativos y acelerados en relación con las TIC, que alterarán de manera considerable la naturaleza y organización del trabajo en las empresas, además de hacer posibles nuevas formas de trabajo y situaciones laborales.

A pesar de que se ha dicho que la introducción de nuevas tecnologías en la empresa puede llevar consigo la destrucción de empleo, hay muchas voces optimistas que piensan que ello se compensaría con la creación de otros nuevos puestos de trabajo. Evidentemente, habrá importantes cambios en la naturaleza del trabajo y en la distribución del empleo entre sectores, lo que exigirá una especial atención a la 
prevención de riesgos en los mismos. El aumento de las posibilidades de teletrabajar o trabajar a distancia cambiará las reglas de juego en una partida donde la producción del accidente de trabajo se produce normalmente en la empresa (un reto más para la regulación de la Seguridad y Salud en el trabajo). Además de que el lugar de trabajo se dispersa, lo mismo ocurre con el tiempo de trabajo. De hecho, la principal regulación comunitaria en materia de prevención de riesgos laborales está relacionada con el tiempo de trabajo.

Desde la perspectiva de la regulación de la prevención de riesgos laborales, los cambios que se prevé puede producir la introducción de las TIC en el trabajo va a poner en cuestión los mecanismos o normas que en la actualidad se han adoptado para la prevención de riesgos. Y es que, la digitalización, si bien permite la reducción o mejor gestión de riesgos laborales, abre la puerta a un mayor número de problemas de seguridad y salud laboral, sobre todo de naturaleza ergonómica, organizativa y psicosocial, que es necesario entender y atender mejor. Habrá de hacerse un uso responsable de la tecnología compatible con la salud laboral.

En cualquier caso, es difícil predecir estos cambios, lo que es seguro es que en materia de prevención de riesgos laborales se plantearán problemas a resolver con:

a) Los nuevos sistemas automatizados, equipos de trabajo y herramientas utilizadas: aunque es posible que la automatización retire a los trabajadores de los entornos peligrosos, también lo es que puedan introducir nuevos riesgos, especialmente influenciados por la transparencia de los algoritmos subyacentes y las interfaces o relaciones trabajador-máquina. Por ello será necesario plantear la prevención "a través del diseño" que integre un enfoque de diseño centrado en el usuario o trabajador y una evaluación avanzada de riesgos laborales de conformidad a las nuevas tecnologías que se integren en el proceso productivo, en el lugar de trabajo, sea este en la empresa o en el domicilio del trabajador (o nómada).

b) La forma de organizar y administrar el trabajo:

- Los factores psicosociales y organizativos serán cada vez más importantes porque las TIC pueden promover cambios en los tipos de trabajos disponibles; el ritmo de trabajo, cómo, dónde y cuándo se realiza; y cómo se gestiona y supervisa.

- El aumento del estrés laboral, especialmente debido al impacto del creciente seguimiento de los trabajadores facilitado por los avances y la mayor ubicuidad de las TIC portables, la disponibilidad 24/7, la confusión de los límites entre la vida laboral y personal, y la economía de las plataformas de internet; ha de evitarse la presión de rendimiento en los trabajadores pues puede llevar consigo el aumento de riesgos profesionales.

- Crecientes riesgos ergonómicos debido al incremento del trabajo por internet y al uso de dispositivos móviles en entornos distintos de la oficina.

- Riesgos asociados a las nuevas interfaces hombre-máquina, especialmente por la ergonomía y la carga cognitiva.

- El incremento del trabajo sedentario, un riesgo asociado a la obesidad y a enfermedades no transmisibles como las cardiovasculares y la diabetes. 
- Riesgos para la ciberseguridad debido al incremento de la interconexión de las cosas y las personas.

- La gestión algorítmica del trabajo y los trabajadores, la Inteligencia Artificial, las tecnologías de seguimiento, como los elementos portables, junto con el Internet de las Cosas y los macrodatos, pueden ocasionar que los trabajadores pierdan el control de sus datos, así como problemas de protección de datos, problemas éticos, desigualdad en la información con respecto a la prevención de riesgos y salud laboral, y presión sobre el rendimiento de los trabajadores.

c) Los modelos, jerarquías y relaciones empresariales y las responsabilidades en materia de gestión de la prevención de riesgos:

- El creciente número de trabajadores considerados (debida o indebidamente) como autónomos y que, en nuestra legislación, quedan fuera de la reglamentación vigente de la LPRL. Por ello es preciso un marco normativo que aclare las responsabilidades en materia de seguridad laboral en relación con los nuevos sistemas y formas de trabajo.

- Cambios en los modelos de negocio y las jerarquías laborales debido al incremento del trabajo en internet y flexible y a la introducción de la gestión algorítmica y la Inteligencia Artificial, que pueden alterar los actuales mecanismos de gestión de la prevención de riesgos laborales.

d) Las características de la fuerza de trabajo y la necesidad de elevar las capacidades, los conocimientos y la información necesarios para trabajar:

- La carencia de las capacidades necesarias en la fuerza de trabajo para utilizar las TIC, adaptarse a los cambios y gestionar el equilibrio entre su vida laboral y personal. Es necesaria la participación de los trabajadores en la aplicación de las estrategias de digitalización y crear un sistema de formación en prevención de riesgos para los trabajadores en el entorno digitalizado.

- Además, se producirán cambios de empleo más frecuentes y una vida laboral más larga. Sería oportuno introducir con claridad en el artículo 25 de la LPRL, referido a la Protección de trabajadores especialmente sensibles a determinados riesgos, la consideración de los trabajadores maduros frente a las nuevas tecnologías.

RIESGOS VINCULADOS A LAS NUEVAS TECNOLOGÍAS Y AUTOMATIZACIÓN DE LOS PROCESOS

\section{Dr. David Montoya Medina}

Universidad de Alicante

I. El cambio de paradigma en la prevención de riesgos laborales: nuevos riesgos asociados a la revolución tecnológica.

II. Riesgos asociados a la automatización y a la robotización.

1. ¿Estamos preparados para interactuar laboralmente con robots?

2. Robots enjaulados y "cobots": ¿aliados en el trabajo o fuente de riesgos? 
III. Un riesgo ya clásico y omnipresente: el trabajo con pantallas de visualización de datos. Particular referencia a los efectos sobre la salud del trabajo informático en periodo nocturno.

IV. El tsunami tecnológico: riesgos asociados a la utilización de las nuevas tecnologías.

1. Tecnología y calidad de vida: ¿resulta completamente inocua la hiperconectividad a la que estamos actualmente expuestos?

2. Dependencia tecnológica, hiperconectividad y trabajo por cuenta ajena: riesgos asociados a la utilización de los recursos tecnológicos en la relación de trabajo.

V. La posición de la empresa como garante de la seguridad y salud de los trabajadores. La necesidad de respuestas adecuadas desde la prevención.

VI. La tímida respuesta del ordenamiento jurídico. El derecho a la desconexión digital: un derecho de naturaleza preventiva instaurado en la ley de protección de datos.

RIESGOS VINCULADOS A LA BIOTECNOLOGÍA

\section{Federico de Montalvo Jääskeläinen}

Profesor Facultad de Derecho ICADE. Miembro Comité Internacional de Bioética de la UNESCO

Es habitual tildar los tiempos que vivimos como tiempos extraordinariamente disruptivos y vincularlo al ingente progreso de la informática y la ingeniería o, en idénticos términos, a la capacidad de superación que se le ofrece ahora al ser humano desde la perspectiva de la electrónica, la mecánica y la computación. Así, la Inteligencia Artificial, la robótica, el Big Data o, más recientemente, el Blockchain se nos presentan como verdaderos impulsores de este cambio o transformación que se muestra como inaudito. La disrupción digital emerge como el fenómeno que marcará inexorablemente el futuro del ser humano. Una mera búsqueda sencilla en internet de la palabra disrupción nos ofrece un ejemplo paradigmático de ello. La disrupción queda en casi todas las entradas que un buscador nos ofrece vinculada a la tecnología o al mundo de lo digital. Las palabras disrupción y digital o tecnológico constituyen el resultado más habitual de dicha búsqueda. De hecho, la propia palabra disrupción parece que fue usada por primera vez para describir los tiempos actuales en un artículo publicado en 1995 en la Harvard Business Review (Disruptive technologies: catching the wave, Joseph L Bower y Clayton M Christensen), vinculada al cambio tecnológico de la empresa, lo que obligaba a los emprendedores a reinventarse.

Sin embargo, desde la perspectiva del impacto en la propia naturaleza e identidad del ser humano, creemos que la verdadera disrupción viene realmente de la mano de la Biología y la Medicina. El verdadero cambio que puede transformar al ser humano de 
manera tal que no encuentra precedente en la Historia nos lo ofrecen las nuevas posibilidades de actuar sobre el propio genoma humano, es decir, sobre el conjunto de genes que constituyen el ADN de cada individuo.

La tecnología nos afecta una vez ya nacidos, pero el avance de la Biología y, en concreto, de la Genética y la Genómica parecen que van a poder determinar nuestro futuro desde nuestros primeros días de vida, desde nuestra vida biológica previa, incluso, al propio nacimiento. En este complejo futuro que se nos predice, no solo se nos podrá superar en nuestras capacidades intelectuales o físicas, incorporando a nuestro entorno y a nuestro propio cuerpo un ingente aparataje tecnológico, sino que se nos podrá mejorar, y ello, incluso, antes de nacer, interviniendo directamente sobre el embrión. La naturaleza de lo humano se pone en cuestión, sobre todo, cuando la alteramos en su propia esencia, no solo en su entorno, y, además, desde su propio inicio.

A este panorama disruptivo no son ajenas las relaciones laborales. En concreto, desde la perspectiva del avance de la genética y genómica surgen nuevos retos para el Derecho en el ámbito de la relación empresario-trabajador, sobre todo, en lo que se refiere al deber de aquél de promoción y protección de la salud del trabajador y la medicina predictiva (test genéticos).

RIESGOS PSICOSOCIALES VINCULADOS A LA SOCIEDAD TECNOLÓGICA

Dr. Juan José Fernández Domínguez

Universidad de León

Los riesgos psicosociales que pueda sufrir la persona del trabajador con origen en una sociedad tecnológica, es decir, "extralaborales" con incidencia o proyección en el trabajo, obliga a reflexionar sobre el documento del Comité Mixto OIT-OMS sobre Medicina de Trabajo. Sopesar, por ende, las interacciones entre el trabajo, su medio ambiente y la satisfacción en el empleo, por una parte; por la otra, las capacidades del empleado, sus necesidades, cultura y situación personal fuera del trabajo.

Esta implicación, lejos de doble, llega o puede llegar a ser triple, pues a los factores laborales y externos al trabajo procederá unir, de manera obligatoria, los individuales de cada persona, para así dar lugar a un cúmulo de determinantes que puede admitir análisis instrumentales en función del factor que quiera ponderarse.

De este modo, por ejemplo, y de tomar en consideración el cuerpo y el anhelo a responder a un arquetipo físico, podrá ponderarse el aspecto personal como un factor de riesgo psicosocial, vinculándolo a exigencias laborales de responder a una imagen (incluyendo la indumentaria); por ejemplo, condicionado a discriminación por razón de obesidad que conduzca a anorexia o bulimia o, en el otro extremo, el intento de alcanzar la excelencia que lleva a la vigorexia. Igual sucedería si el elemento de medir se sitúa en el afán de lograr la integración social en determinados entornos culturales, 
donde el consumo de alcohol y drogas puede llevar a riesgos de alcoholismo y drogodependencia.

Pero si la tecnología es la protagonista, la dependencia más allá de la que, en su caso, procederá utilizar en el trabajo (o como proyección continuada del trabajo, en el caso de workaholism e, incluso, del work engagement) es sin duda, el supuesto más documentado. Junto a la dependencia, las situaciones de ciberacoso o, sin llegar a tal, el sentimiento de vigilancia y control tecnológico, cuando no de abierta tecnofobia o incapacidad de adaptarse a contextos sociales donde muchas actividades no presentan alternativas al recurso a la tecnología que, por una u otra causa, puede acabar siendo fuente de estrés.

Cabrá profundizar en cualquiera de esos tres, y hasta perfilarlos en función de los ingresos familiares, los niveles educativos, el canon de igualdad (género, raza, religión, discapacidad, etc.), el entorno social (incluido salud, vivienda, transporte) o las condiciones de vida; es decir, situarlos en un ámbito en el cual la tecnología (riesgo en sí misma) acaba alcanzando un índice más trascendente a partir de su contexto social.

Habrán de ponerse en conexión, en fin, con cuanto no deja de ser sino un efecto directo de la tecnología, que toma la falta de apoyo social para hacer quebrar el modelo demanda-control, y hacer que las situaciones de necesidad se agraven cuando falta el sostén de las personas, al haber trocado lo emocional por lo tecnológico.

\section{RIESGOS VINCULADOS A LAS FUENTES DE ENERGÍA RENOVABLES Y RECICLAJE}

\section{Dr. Javier Fernández-Costales Muñiz}

Universidad de León

La gestión de residuos es una actividad necesaria, que comporta riesgos para el medio ambiente $\mathrm{y}$, sobre todo, para los trabajadores. La recogida y gestión de residuos no está exenta de accidentes fatales, que ocurren con relativa frecuencia.

El sector de la gestión y tratamiento de residuos presenta actualmente una gran importancia debido, fundamentalmente, al establecimiento de disposiciones legislativas, tanto nacionales como autonómicas, derivadas de la aplicación de la política de desarrollo sostenible establecida por la Unión Europea para una adecuada protección de la salud y del medio ambiente. Todo ello sin olvidar la sensibilización social existente sobre todos los aspectos relacionados con la protección del medio ambiente.

La Directiva 2008/98/CE establece el marco jurídico de la Unión Europea para la gestión de residuos y proporciona los instrumentos que permiten disociar la relación existente entre crecimiento económico y producción de residuos, haciendo especial hincapié en la prevención, entendida como el conjunto de medidas adoptadas antes de 
que un producto se convierta en residuo, para reducir tanto la cantidad y contenido de sustancias peligrosas como los impactos adversos sobre la salud humana y el medio ambiente de los residuos generados.

Igualmente, durante los últimos años la energía renovable ha ido adquiriendo en nuestra sociedad un considerable peso debido al impulso que ha ido obteniendo por parte de los distintos gobiernos, así como por la necesidad de buscar otros métodos de alimentación, aparte de los tradicionales como la termoeléctrica nuclear o la termoeléctrica clásica. La generación de energía eléctrica mediante agentes no contaminantes como son las energías eólica, hidráulica, geotérmica o solar, además de contribuir a reducir las emisiones de gases nocivos y la elevada tasa de dependencia de las importaciones, genera una amplia actividad empresarial, dando origen a numerosos empleos, algunos de nuevas características, que a su vez generan diferentes tipos de riesgos para los trabajadores, pues las cada vez más avanzadas tecnologías empleadas y los métodos científicos utilizados suponen nuevas formas de nocividad, riesgos, peligros y factores de afectación para la salud laboral.

España es uno de los primeros países del mundo en cuanto a la implantación de renovables, lo que conlleva también una gran responsabilidad en materia de Prevención de Riesgos Laborales.

RIESGOS VINCULADOS A LAS NUEVAS FORMAS DE TRABAJO AGROPECUARIO Dra. Henar Álvarez Cuesta

Universidad de León

El trabajo agropecuario en España siempre ha revestido de una importancia y trascendencia fundamental y ha adquirido una nueva dimensión con la apuesta por un cultivo y una ganadería ecológica, asumiendo los postulados de la sostenibilidad medioambiental y la lucha contra el cambio climático.

Las características de las actividades agrícolas y ganaderas en el territorio español no son uniformes, sino que aparecen diferenciadas por el tamaño de las explotaciones (vinculado al trabajo por cuenta ajena o propia) y las distintas condiciones climáticas y geográficas.

Para hacer frente a los riesgos provocados por estas actividades, conviene atender a sus diferencias y a las variadas tareas desempeñadas, en las cuales potenciales riesgos pueden quedar resumidos como a continuación se expone:

- En primer lugar, riesgos físicos derivados de la utilización de útiles, instrumentos, vehículos o maquinaria, o de las malas posturas y el esfuerzo reiterado que dicha actividad requiere de forma continuada, todo lo cual puede provocar incidentes de diversa naturaleza como caídas al mismo o distinto nivel, atrapamientos y atropellos, caída de objetos, carga física, ruido y vibraciones, incendios y explosiones, contactos eléctricos, etc. 
- En segundo término, riesgos derivados del inevitable trabajo con animales (riesgos físicos), pero también riesgos biológicos y químicos. El RD 1299/2016, de 10 de noviembre, por el que se aprueba el cuadro de enfermedades profesionales en el sistema de la Seguridad Social y se establecen criterios para su notificación y registro, contempla expresamente para los ganaderos las enfermedades infecciosas o parasitarias transmitidas al hombre por los animales o por sus productos y cadáveres (zoonosis) y con manifestaciones como brucelosis, tuberculosis bovina, rabia, tétanos, carbunco, toxoplasmosis, leptopsoriasis, pulmón de granjero, tularemia, fiebres, gripes, etc., así como las causadas por la inhalación de sustancias y agentes no contemplados de forma expresa en otros apartados.

Respecto al riesgo químico, este deriva del uso de pesticidas y abonos en el trabajo agrario. Aparece regulado en el RD 374/2001, de 6 de abril, sobre la protección de la salud y seguridad de los trabajadores contra los riesgos relacionados con los agentes químicos durante el trabajo, y puede derivar de la utilización de plaguicidas y fertilizantes, abonos orgánicos, medicamentos veterinarios y productos zoosanitarios, fitosanitarios o biocidas, sustancias toxicas, causticas y corrosivas, gases, etc.

- Riesgos ambientales, especialmente los relativos al trabajo al aire libre o en locales no cerrados bajo condiciones climatológicas extremas de temperatura y humedad.

- Sin olvidar en este elenco los riesgos psicosociales, que pueden generar estrés, ansiedad o la depresión.

\section{RIESGOS VINCULADOS A LA CONSTRUCCIÓN ECOLÓGICA}

\section{Dra. Susana Rodríguez Escanciano}

Universidad de León

Siendo muy difícil establecer una línea divisoria entre riesgo profesional y riesgo ecológico al ser dos variables directamente imbricadas, el sector de la construcción ha tenido la virtud de actualizarse y adaptarse a la transformación operada desde una agotada edificación expansiva e invasiva hacia una actividad empresarial más sostenible desde el punto de vista de la salubridad laboral y desde la perspectiva de la calidad atmosférica. Cuando está bien planificado, el crecimiento urbano encierra el potencial de generar ocupaciones laborales menos penosas en el marco de una rama industrial basada en la renovación, regeneración y rehabilitación integral de los edificios, incorporando materiales menos contaminantes, adelantados sistemas bioclimáticos $\mathrm{y}$, cómo no, modernas herramientas, que permiten mejorar la seguridad de los trabajadores. Ahora bien, tampoco cabe dar saltos en el vacío y olvidar que los cambios en el diseño de la actividad urbanística no eliminan de radice los riesgos laborales, que ciertamente pueden ser distintos, en ocasiones, a los de su configuración precedente, pero no por ello son menos importantes, no en vano "construcción verde" no es sinónimo de ausencia de peligro, realidad que exige seguir adoptando como máxima una cultura preventiva. 
La construcción, en general y la ecológica en particular, integra uno de los sectores de la actividad con mayores tasas de accidentabilidad del tejido económico, generando riesgos ciertos, unos heredados del pasado (trabajos en altura, resbalones, tropiezos, cortes, caídas, vuelco de maquinaria, sepultamientos, ruido, vibraciones, atropellos, sobreesfuerzos, posturas difíciles, cargas, golpes con objetos grandes, inclemencias meteorológicas, explosiones, incendios, impactos eléctricos, almacenamiento y manipulación de productos químicos, espacios confinados, destajos, envejecimiento de los trabajadores...), y otros de nueva gestación, derivados de novedosos materiales, tecnologías y diseños en las obras que permiten una mayor sostenibilidad del entorno natural mediante la reducción del consumo de energía y materias primas, una disminución de gases de efecto invernadero y una minimización y mayor aprovechamiento de los residuos a través de su reutilización, reciclaje o valorización, si bien, al tiempo, provocan el surgimiento de nuevas situaciones negativas, tales como la instalación de tecnologías de energía renovable en altura de difícil manejo o la carga de residuos hasta los contenedores, combinadas con la utilización de nuevos aislantes o productos que contienen nanomateriales o con exposiciones incontroladas a fibras, a partículas ultrafinas o a microorganismos desconocidos, unidas al uso de elementos reciclados, reutilizados o renovables con altas dosis de alérgenos, sustancias cancerígenas (bambú, paja, lana de oveja, lino, corcho, papel, disolventes, escoria fundida, feldespatos, titanio de potasio, cenizas volantes, sílice cristalina, fibra cerámica, roca, alquitrán de hulla, madera, resinas, serrín, cenizas de carbón...) o de productos acuosos que contienen biocidas, así como de fuentes de energía alternativa (solar pasiva, eólica y bioenergía), cuya obtención presenta riesgos laborales de incendio o de electrocución. Es más, los edificios verdes suelen ser más herméticos y estar perfectamente aislados para ahorrar energía, de modo que la ventilación suele ser reducida durante las labores interiores de acabado, lo cual puede aumentar la exposición a compuestos orgánicos volátiles de pinturas o adhesivos y al polvo, en particular, la sílice cristalina, serrín, cenizas de carbón, fibra cerámica, roca, mezclas bituminosas que contienen alquitrán de hulla, arcilla, mercurio, lana de vidrio, siliconas, aceites, disolventes, yesos, desencofrantes, humos de escape de motores diesel o espuma de poliuretano, exposiciones todas estas que pueden causar dermatitis, irritación ocular, enfermedades en las vías respiratorias, de las membranas mucosas de los ojos y del tracto intestinal, así como daños al hígado y a los conductos biliares. En fin, y por no seguir, es alta también la incidencia de las radiaciones UV entre trabajadores que realizan sus tareas en el exterior y pasan gran parte de su jornada laboral expuestos.

RIESGOS VINCULADOS A LAS NUEVAS FORMAS DE MOVILIDAD SOSTENIBLE Dr. Rodrigo Tascón López Universidad de León

Los increíbles avances tecnológicos que está contemplando la humanidad tienen influencias múltiples sobre los más variados aspectos de la vida social y jurídica. Uno entre muchos es el relativo al desplazamiento de trabajadores, esto es, la necesidad que tienen los operarios de desplazarse, moverse, en el mundo físico, para desarrollar 
su prestación profesional, ya sea de forma normal o habitual; ya de forma más excepcional, para realizar tareas en lugares distintos al centro de trabajo (viajes en misión).

El Derecho del Trabajo se ha ocupado tradicionalmente de estas cuestiones desde varios puntos de vista: limitaciones a la decisión temporal de desplazamiento de trabajadores y a la definitiva de la movilidad geográfica (art. 40 ET); consideración de accidente de trabajo tanto el sufrido in itinere como el ocurrido en misión (art. 156 LGSS). La industria 4.0 influye, al menos, de dos formas diferentes en los desplazamientos de los trabajadores:

$1^{\circ}$. La tecnología evita y evitará desplazamientos de los trabajadores: las nuevas herramientas tecnológicas están empequeñeciendo el mundo. Hoy se puede en tiempo real tener una reunión virtual con un cliente o proveedor, enviar gran cantidad de información en cualquier lugar del mundo, perfeccionar contratos a través de firma electrónica, trabajar a distancia (algo que se ha facilitado tras la reforma del art. 13 ET operada por la famosa reforma laboral de 2012, que no todo hizo mal...) haciendo llegar el fruto del esfuerzo a la empresa en el mismo momento en el que es realizado por el teletrabajador que, a su vez, puede ser controlado perfectamente por la empresa a través de dispositivos remotos (que, en principio, y si han sido debidamente informados, son lícitos y no atentan contra derecho fundamental inespecífico alguno)...

Todas estas potencialidades han de llevar a una disminución de los desplazamientos de trabajo porque es un objetivo deseable desde diversos puntos de vista: tanto para el trabajador individual (las limitaciones del art. 40 muestran a las claras que el interés del operario es precisamente no tener que viajar por motivos laborales) para facilitar la conciliación de la vida personal, familiar y laboral; como colectivo (menor impacto ambiental, reducción de $\operatorname{atascos} .$.$) .$

$2^{\circ}$. La tecnología permite nuevas formas de desplazamiento más rápidas y seguras. $\mathrm{Y}$ esto puede ser usado por los trabajadores y favorecido y potenciado por las empresas como vía para prevenir posibles siniestros, evitando así responsabilidades civiles privadas y públicas de Seguridad Social (lo que redunda en beneficio de las maltrechas arcas del Sistema).

Por otro lado, el Derecho de la Seguridad Social, encargado de prestar protección a quien sufra un accidente como consecuencia de un desplazamiento profesional, ha experimentado (está experimentando) una constante reconstrucción del concepto de accidente de trabajo, consagrado en el art. 156 LGSS, para adaptarlo a las nuevas realidades.

Así, el accidente in itinere, aun cuando sigue requiriendo de sus característicos elementos locativo, teleológico y medial, ha sido flexibilizado en los últimos tiempos por la jurisprudencia para entender, conforme a la realidad social, que se puede compaginar un domicilio familiar con otro profesional, al que viajar la noche antes de la incorporación efectiva al trabajo, quedando el accidente ocurrido en dicho trayecto incorporado al concepto de contingencia profesional. De igual forma, se han aceptado 
como medios idóneos de desplazamiento aquellos novedosos derivados del nuevo contexto social, como, por ejemplo, acudir al trabajo en patinete eléctrico.

Por su parte, el accidente en misión ha sido considerado plenamente por la jurisprudencia como accidente de trabajo, aun cuando los pronunciamientos más recientes han explicado que no pueden extenderse sus efectos a los períodos en los que el trabajador estaba disfrutando de períodos de descanso en el hotel o de momentos de ocio en playas o lugares similares, salvo que se pruebe de forma indubitada su relación con el trabajo.

\section{LA FORMACIÓN INICIAL EN MATERIA PREVENTIVA PARA TRABAJADORES JÓVENES Y PARA LOS INMIGRANTES CON ESCASA EXPERIENCIA LABORAL \\ Dr. Roberto Fernández Fernández Universidad de León}

La formación en materia preventiva dentro de la empresa se viene conformando como una de las grandes preocupaciones para todos los partícipes en el sistema español de relaciones laborales, pues una adecuada política en esta materia permitirá cumplir con mayor grado de optimización las obligaciones preventivas que tienen tanto el empresario (fundamentalmente este) como el trabajador.

En este sentido, la obligación empresarial recogida en el artículo 19 LPRL de garantizar al trabajador la formación teórica y práctica, suficiente y adecuada, en materia preventiva para hacer frente a los riesgos en el trabajo con una mínima pericia y eficacia se conforma como uno de los quehaceres más importantes para garantizar la seguridad y salud del trabajador.

De ahí la trascendencia que la formación inicial del trabajador ha de jugar en este ámbito, más aún para determinados grupos de empleados que pueden estar necesitados de una protección específica al respecto, pues manifiestan más probabilidades de sufrir algún tipo de lesión que otros trabajadores debido a sus características personales, profesionales o sus condiciones de trabajo.

En tal situación se pueden encontrar los trabajadores jóvenes y los inmigrantes con escasa experiencia profesional, cuya falta de pericia puede constituir un elemento a tomar en consideración a la hora de diseñar y planificar la formación que estas personas deben recibir para realizar su prestación de servicios con las suficientes garantías de seguridad.

A partir de la premisa anterior, se van a explorar las posibles adaptaciones o sugerencias que la formación en materia preventiva de estos colectivos puede necesitar con el fin de asegurar su salud en el trabajo. 
LA NECESIDAD DE RECUALIFICACIÓN EN MATERIA PREVENTIVA DE LOS TRABAJADORES MADUROS

Dra. María de los Reyes Martínez Barroso

Universidad de León

Para hacer posible y viable una auténtica "cultura de la seguridad" en el seno de la empresa es preciso apostar por una colaboración activa en la materia, como manifestación expresa en materia de responsabilidad social, que requiere una apuesta mayor por una inversión económica en medios materiales y en procesos formativos, además de otras actuaciones concretas dirigidas a colectivos específicos. A continuación se da cuenta de una serie de líneas de actuación relacionadas con las condiciones de trabajo de los mayores de 55 años, con particular atención a la seguridad y la salud en el trabajo.

\section{Reducción de la jornada laboral}

En España, la VI Encuesta Nacional de Condiciones de Trabajo refleja una gran rigidez en los horarios del colectivo de trabajadores de entre 55 y 64 años, lo cual, unido al hecho de que la influencia del trabajo en la salud se acentúa en los mayores de 55 años, obliga a considerar que una mayor flexibilidad en la organización del trabajo permitiría a este colectivo retirarse progresivamente de la población activa reduciendo su jornada laboral y evitando su cese total. Desde una perspectiva integrada, el dilema entre cese o prolongación de la actividad admite, al menos, modulaciones en dos criterios. En primer término, la actividad puede seguir, pero con reducción del tiempo de actividad y como el tiempo de trabajo es, por naturaleza, una condición muy flexible, esa reducción admite diversas fórmulas: jornada a tiempo parcial, semana de cuatro días, aumento de los días de descanso en el ciclo anual, etc. En segundo lugar, puede cambiar la función o el contenido de la prestación, dando prioridad a la actividad de asesoría y de consejo, de formación de los jóvenes y nuevos empleados, dedicación a tareas relacionadas con la clientela o, en fin, participación en equipos de trabajo interdisciplinares con distintos conocimientos y experiencias pues, lo que cuenta son las capacidades, no las incapacidades. Es más, el reparto de trabajo puede intentarse también utilizando el tiempo de trabajo, pero no actuando sobre la jornada, sino sobre el tiempo de trabajo completo de la vida de los trabajadores. Por otra parte, a nivel empresarial y en el marco de las condiciones de trabajo, se pueden impulsar medidas dirigidas al desarrollo de a) Nuevas competencias y destrezas en el trabajo, a través de la rotación de tareas o del enriquecimiento del puesto. b) Estrategias organizativas que adapten los ritmos, descansos, turnos y horarios de trabajo a las condiciones físicas del trabajador mayor y medidas que aborden cambios en la actividad. c) Programas complementarios encaminados a mantener actualizados los conocimientos técnicos a nivel profesional (cualificaciones de adaptación).

\section{Evaluación de riesgos, vigilancia de la salud, formación e información para la seguridad y salud en el trabajo de los empleados de más edad.}

De acuerdo con los datos de la Encuesta Nacional de la Salud (MSCPS), a partir de los 55 años, la población es más vulnerable a una serie de procesos patológicos 
directamente relacionados con el propio envejecimiento y las patologías degenerativas consustanciales a aquel y reflejo, además, del efecto acumulado a largo plazo de los estilos de vida poco saludables (alta prevalencia de artropatías degenerativas, hipertensión, etc.). Una persona que lleva realizando las mismas funciones durante mucho tiempo genera una monotonía en su desempeño que puede tener consecuencias negativas en su salud. A partir de tal constatación resulta necesario explorar vías de actuación como las siguientes: a) Incidir en la importancia de contemplar en las evaluaciones de riesgo los factores inherentes a la edad y a la antigüedad. b) Proceder (a partir de los resultados de tal evaluación) a una adecuada planificación de la actividad preventiva, que incluya, con carácter prioritario, un programa adecuado de vigilancia de la salud específica en función de los riesgos y adaptada a la edad del trabajador, en la que se tengan en cuenta, entre otros, factores tales como la movilidad limitada de las articulaciones; la disminución de la fuerza; la reducción de la capacidad funcional física, de la percepción y de la capacidad para tomar decisiones; la reducción de la capacidad visual y auditiva; la ralentización de la capacidad de reacción. Dicho "programa de salud" debería comprender, asimismo, actividades formativas e informativas: a) Formación específica a los trabajadores mayores sobre cómo mantener y promover su propio rendimiento físico y psicológico a través de estrategias acordes a las exigencias del trabajo (gestión del estrés, prevención del agotamiento); b) Información sobre cómo conseguir un buen mantenimiento físico: promoción de buenos hábitos de salud (nutrición o recomendación de prácticas deportivas). Dichas evaluaciones de riesgos y la planificación y programación de la actividad preventiva consecuencia de la misma deben tener en cuenta, asimismo, los riesgos específicos que afecten a las mujeres de más edad.

\section{Movilidad funcional por razones de seguridad y salud en el trabajo}

La finalidad primordial de la movilidad funcional es la búsqueda permanente de la mejor adecuación entre el puesto de trabajo y las aptitudes profesionales del trabajador. Por medio de la adaptación de las condiciones de trabajo se protege la capacidad, se previene la pérdida de salud y se facilita la autonomía personal y, en este sentido, la formación incide directamente sobre la prestación en sí, las funciones y las tareas que se desempeñan. En otros términos, el cambio de condiciones de trabajo afecta a la periferia de la prestación laboral y remite a lo que puede denominarse "calidad de vida laboral". Si de la evaluación de riesgos se concluyera, a pesar de llevar a cabo todas las medidas reflejadas en el apartado anterior, que la edad pudiera comenzar a ser un hándicap para que el trabajador desempeñe determinados puestos de trabajo, deberán plantearse todas las opciones posibles, antes de su salida del mercado de trabajo. Entre ellas, como medida preventiva, la posibilidad de aplicar medidas de flexibilidad interna en la empresa, incluyendo el cambio de puesto de trabajo, junto con otras estrategias que, desde un enfoque integral, estén dirigidas a la adaptación del puesto de trabajo con el fin de garantizar la seguridad y salud del trabajador.

\section{Medidas en el marco de la investigación encaminadas a la protección social}

En este ámbito resulta necesario impulsar la realización de investigaciones específicas en materia de condiciones de trabajo y salud de los trabajadores mayores que permitan 
detectar perfiles de morbilidad asociados con la edad y ayuden al diseño de intervenciones concretas, así como la realización de estudios epidemiológicos que determinen aquellas ocupaciones potencialmente más peligrosas en función de la siniestralidad y definan el impacto que, sobre la misma, ejerce la edad.

\section{LAS MUJERES ANTE LA BRECHA DIGITAL: ESPECIAL ATENCIÓN A LOS} RIESGOS EN MATERIA PREVENTIVA

\section{Dra. Beatriz Agra Viforcos}

Universidad de León

1. Nuevas tecnologías y trabajo:

- La Industria 4.0.

- Impacto de robótica y digitalización el trabajo y el empleo:

○ Procesos de destrucción y creación de empleo.

○ Trabajo 4.0.

○ Nuevos escenarios para la seguridad y salud en el trabajo.

2. Nuevas tecnologías y prevención de riesgos laborales:

- Nuevas tecnologías y protección del trabajador frente al riesgo laboral.

- Riesgos laborales derivados de las nuevas tecnologías. En especial, riesgos psicosociales y, en concreto, tecnoestrés:

○ Modalidades: tecnoadicción, tecnofatiga y tecnoansiedad.

- Demandas del trabajo con TICs y tecnorecursos laborales y personales.

- Estrategias de intervención preventiva en relación con el tecnoestrés.

3. Brecha digital por razón de género:

- Brecha digital de acceso y aprovechamiento.

- Cuestión formativa: carácter minoritario de las mujeres en los estudios de STEM (los más demandados).

$\circ$ Estudios universitarios.

○ Formación profesional.

- Escasez de mujeres en sectores tecnológicos y punteros (y peores condiciones retributivas).

4. Conclusión: brecha digital y prevención de riesgos. 
LA POTENCIALIDAD DE LOS CAMBIOS TECNOLÓGICOS PARA LA INCORPORACIÓN SEGURA AL MERCADO DE TRABAJO DE LAS PERSONAS CON DISCAPACIDAD

\section{Dr. José Gustavo Quirós Hidalgo}

Universidad de León

Durante los últimos años, la contratación de las personas con discapacidad mantiene su tendencia al alza alcanzando a finales del 2018 el mejor registro de toda la serie histórica, aumentando más del $100 \%$ en la última década, si bien ese incremento se ha producido en su mayoría en el ámbito protegido de los Centros Especiales de Empleo.

Los anteriores datos acreditan, en primer lugar, que las nuevas tecnologías son un elemento fundamental y con un impacto creciente para fomentar la contratación de las personas con discapacidad en el mercado laboral ordinario, pues favorecen la integración y la empleabilidad mediante la formación, la visibilidad del perfil profesional, el acceso a las ofertas de empleo, el desarrollo del trabajo en mejores condiciones, la reducción de barreras y la equiparación de capacidades.

En segundo término, también cabe constatar un aumento del teletrabajo, o la opción por el mismo, en el colectivo de las personas con discapacidad, si bien se mantiene en niveles muy bajos y que ponen de manifiesto la cultura del presentismo laboral que aun sigue primando. El teletrabajo supone una alternativa real, e incluso una garantía, para que aquellas personas con movilidad reducida no queden excluidas del mercado laboral, ofreciendo ventajas para todas las partes implicadas.

Como tercera reflexión, las redes sociales se han convertido en una ventana al mundo y en una herramienta que atenúa las debilidades y potencia las fortalezas, donde las personas con discapacidad pueden actuar en igualdad de condiciones. En este sentido, los estudios demuestran que el uso de estas redes sociales aumentan las posibilidades de encontrar empleo.

Por otra parte, también es posible que la existencia de las nuevas tecnologías y su disponibilidad o posibilidad de uso real y eficaz para la persona con discapacidad determine nuevas barreras difíciles de sortear, como por ejemplo las relacionadas con las opciones de accesibilidad, la ergonomía o su propio funcionamiento, lo cual se puede traducir en una limitación muy importante especialmente en la búsqueda de empleo, pues un gran porcentaje de las ofertas son publicadas en plataformas digitales.

En definitiva, la tecnología tiene todavía mucho por explorar y nuevas acciones que poner en marcha para seguir impactando de manera positiva en la vida de todos, en especial de las personas con discapacidad. Por ejemplo, soluciones protésicas más avanzadas para las personas con discapacidad física; avances y uso de la Realidad Virtual Inmersiva para reducir el dolor crónico; transporte más accesible orientado a la independencia de los que lo tienen más difícil; mejoras en materia de inteligencia artificial para lograr una mayor seguridad ciudadana y bienestar a todas las personas 
con discapacidad; perfección de los sistemas de coordinación ojo-mano para que la tecnología haga llegar órdenes enviadas mediante la vista a las manos en casos de paraplejias y tetraplejias; o, por no seguir, por ejemplo, abaratamiento de las soluciones tecnológicas o, en su defecto, el aumento de ayudas económicas que faciliten su compra.

MESA REDONDA: Soluciones para un adecuado diseño de la prevención de riesgos en el talento sin fronteras y las nuevas vías de emprendimiento PREVENCIÓN DE RIESGOS Y TALENTO SIN FRONTERAS

\section{Dra. Natalia Ordóñez Pascua}

Universidad de León

El fenómeno de internacionalización de la actividad empresarial y profesional ha supuesto la proliferación de entidades que aumentan su producción $\mathrm{u}$ ofrecen sus servicios fuera de sus fronteras nacionales e incrementan de manera notable los supuestos de movilidad internacional de los trabajadores que tienen a su servicio.

Fuera de las tradicionales multinacionales que disponen ya de políticas de expatriación arraigadas, aparecen una multitud de entes productivos que necesitan de una gestión adecuada -entre otros y por cuanto aquí importa- en materia de prevención de riesgos laborales de aquellos trabajadores que van a ejercer su actividad en el extranjero por un desplazamiento temporal de mayor o menor duración.

Proceder a la gestión de los trabajadores desplazados es unos de los grandes retos que afrontan las compañías en los procesos de internacionalización La situación normativa muestra una mayor claridad, aún con sus luces y sombras, cuando la actividad laboral es desarrollada en aquellos países que forman parte de la Unión Europea, para los cuales existen reglas y protocolos de aplicación común establecidos fundamentalmente en la Directiva 96/71, de 16 de diciembre, sobre el desplazamiento de trabajadores efectuado en el marco de una prestación de servicios cuya transposición al ordenamiento español se produjo mediante la Ley 45/1999, de 29 de noviembre, sobre el desplazamiento de trabajadores en el marco de una prestación de servicios transnacional. Posteriormente, la norma europea ha sido modificada por la Directiva 2018/957 de 28 de junio.

Sin embargo, supone una dificultad añadida el establecimiento y control de actividades preventivas en las situaciones de desplazamientos de trabajadores a terceros países con legislaciones muy dispares a la nacional de origen del empleado y en los cuales no existe legislación específica, teniendo que acudir a los convenios internacionales vigentes.

En principio, tratándose de un desplazamiento temporal efectuado por una empresa establecida en España, las normas de competencia determinarían como ley objetivamente aplicable la nacional del trabajador, así como competencia a los juzgados y tribunales nacionales en orden a conocer de las pretensiones derivadas del 
contrato de trabajo, y de aquellas cuestiones litigiosas relativas a la seguridad y salud en el trabajo. Además, la empresa debería ser capaz de garantizar a sus trabajadores desplazados aquellas condiciones mínimas de protección establecidas en el ordenamiento jurídico del país de destino.

En este contexto, la gestión de prevención de riesgos laborales del trabajador transnacional debería ser contemplada en dos momentos diferentes: en primer lugar, antes de proceder al desplazamiento, donde se tratará de evaluar las condiciones del país de destino y las necesidades concretas que el trabajador puede tener cuando llegue al país de recepción; en segundo, durante su estancia, en donde será necesario satisfacer las necesidades diarias que en materia preventiva puedan surgir.

LA PREVENCIÓN DE RIESGOS EN TRABAJADORES DESPLAZADOS

\section{F. Xabiere Gómez García}

Universidad de León

El proceso de liberalización de los flujos de capital entre los distintos Estados del planeta - conocido como globalización económica- no ha producido realmente una mundialización de los mercados de trabajo; sin embargo, sí que ha difuminado las fronteras físicas y geográficas en lo que se refiere a la formación de cadenas de valor multinacionales y a la internalización de las empresas mediante el traslado de partes de la misma o el establecimiento de centros productivos en otras zonas fuera del Estado de origen. Esta nueva coyuntura ha resultado en un incremento de los desplazamientos migratorios internacionales, con la novedad de que ahora ya no solo se produce una movilidad «por el empleo», sino también «en el empleo», por cuanto las personas trabajadoras cumplen, cada vez más, misiones temporales comprometidas por su empleador en Estados distintos al del inicio de la relación laboral. Además, los intensos cambios tecnológicos, que posibilitan la reducción del coste de estos desplazamientos, y los efectos de la más reciente crisis económica han implicado su extensión desde los perfiles cualificados o especializados hacia el resto de empleos.

A pesar del citado aumento y su paulatina importancia, la figura del expatriado en el marco de un contrato de trabajo presenta una insuficiente consideración legal, lo que dificulta la aplicación, entre otras, de las normas de prevención de riesgos, habida cuenta que en ocasiones dicho contrato se rige por la normativa nacional pero la protección del trabajador tiene que prestarse, necesariamente, en el país de destino o, forzosamente, con la intervención tanto del país de origen como del país de destino. Por el contrario, algunos convenios colectivos sí establecen un régimen jurídico propio, aunque la mayor atención a estas situaciones de movilidad internacional aparece en los instrumentos internos empresariales, por ejemplo, a través del típico Manual del Expatriado.

Desde el ámbito de la prevención de riesgos laborales debe tenerse en cuenta que, a los riesgos habituales de la actividad profesional desempeñada, deben añadirse 
aquellos propios del país de destino, aunque no siempre será fácil para la empresa prever los posibles riesgos al objeto de extremar la protección del trabajador desplazado; como tampoco el comportamiento de este último, no solo ante los riesgos profesionales, sino ante los riesgos vitales que se pueden presentar en ese país. En este escenario, son ineludibles cuestiones como las situaciones de conflicto social, la seguridad vial, la vacunación sanitaria, las condiciones naturales y meteorológicas y, en particular, la influencia de los riesgos psicosociales.

Con independencia de las discrepancias en relación a la ley aplicable o al foro de competencia judicial, los derechos y obligaciones en materia preventiva deben de estar garantizados. Puesto que el mero cumplimiento de las normas nacionales de prevención de riesgos laborales no garantiza la exención de responsabilidad empresarial, cobra especial relevancia la adecuada diligencia en materia de información periódica, formación, evaluación de riesgos, coordinación de actividades empresariales (en su caso) o la vigilancia periódica de la salud.

SITUACIÓN DE LA PREVENCIÓN LABORAL EN EL EMPRENDIMIENTO DIGITAL Dña. Cristina González Vidales

Universidad de León

El empleo es uno de los elementos fundamentales que tienen los estados para crecer económicamente y mejorar la calidad de vida de los ciudadanos, por ello, la Unión Europea ha defendido desde el Tratado de la Unión Europea (TUE), artículo 3, el pleno empleo como un objetivo para alcanzar un desarrollo económico sostenible. Con esta declaración de intenciones, la Estrategia 2020, reconoce la importancia del emprendimiento y la necesidad de fomentar el espíritu emprendedor para generar empleo, haciendo referencia al emprendimiento en varias ocasiones, y asociándolo a la innovación y al crecimiento económico. Por otra parte, desde la Unión se ha identificado claramente emprendimiento con trabajo por cuenta propia como se puede comprobar en la Comunicación de la Comisión «Hacia una recuperación generadora de empleo».

En cuanto a los trabajadores en plataformas digitales, la doctrina iuslaboralista se encuentra inmersa en el debate acerca de la calificación de las personas que prestan servicios en el marco de la gig economy bien como trabajadores por cuenta ajena bien como trabajadores por cuenta propia. Una disyuntiva que genera una clara inseguridad jurídica, en especial, para los prestadores de servicios, y sobre el que los tribunales están empezando a pronunciarse. La calificación de estos trabajadores como autónomos o trabajador asalariado supondrá, como se podrá comprobar seguidamente, un mayor o menor protección en materia preventiva.

La Ley 31/1995, de 8 de noviembre, de prevención de Riesgos Laborales (LPRL) verdadero cuerpo central y básico del ordenamiento preventivo y que regula la protección de la seguridad y salud en el trabajo de los trabajadores (asalariados), ya estén vinculados por un contrato de trabajo o bien por una relación administrativa de 
prestación de servicios (funcionarios públicos), establece que el «trabajo realizado por cuenta propia», no estará sometido a la legislación preventiva, excepto en aquellos aspectos que por precepto legal se disponga expresamente, debiendo cumplir con los «derechos y obligaciones que puedan derivarse para los trabajadores autónomos» (art. 3.1. LPRL). De ello se deduce que la norma no comprende un derecho general del trabajador autónomo a la protección de la seguridad y de la salud en el desempeño de su trabajo.

La LPRL alude explícitamente a los trabajadores autónomos solamente en dos artículos 15.5 y 24. Así, por un lado, en el artículo 15.5 se determina que los trabajadores autónomos pueden concertar operaciones de seguro para garantizar como ámbito de cobertura la previsión de riesgos derivados del trabajo. Y, por otro lado, el artículo 24 se refiere a los trabajadores autónomos en el marco de los derechos y deberes en materia de coordinación de actividades empresariales, precepto este que ha sido desarrollado mediante el Real Decreto 171/2004, de 30 de enero, por el que se desarrolla el artículo 24 de la Ley 31/1995, de 8 de noviembre, de Prevención de Riesgos Laborales, en materia de coordinación de actividades empresariales. Y es, en concreto, en este último artículo en el que se incluyen los derechos y obligaciones de los trabajadores autónomos; eso sí, circunscritos exclusivamente al supuesto de coordinación de actividades empresariales. 